\title{
Effects of Liquid Impurity on Laser-Induced Gas Breakdown in Quiescent Gas: Experimental and Numerical Investigations
}

\author{
T. Ukai, H. Zare-Behtash, C. White, and K. Kontis
}

\begin{abstract}
This paper reports that the presence of liquid impurity significantly affects the shock wave structure induced by laser-induced gas breakdown in a quiescent gas. A spherical blast wave is formed when there are no suspended liquid particles, whereas an elliptic shaped blast wave appears in gas breakdown with the presence of suspended liquid particles. The elliptic shaped blast wave has a higher overpressure magnitude on the perpendicular axis of the laser path.
\end{abstract}

\section{Introduction}

Laser-induced gas breakdown has the ability to change the flow characteristics due to rapid local heating. The local high temperature induces the generation of plasma and a spherical blast wave, and then a vortex ring is formed due to the asymmetric shape of the plasma region in the laser focal position [1]. According to both numerical and experimental investigations of laser energy addition ahead of a bow shock wave [2,3], the high-temperature region and the vortex ring both contribute to a drag reduction. Therefore, the laser-induced gas breakdown can be used in engineering applications such as flow control [4] as well as laser plasma igniters [5].

Laser-induced gas breakdown for engineering applications is not necessarily used in an ideal gas without any impurities. The laser beam may interact with atmospheric aerosols including liquid and solid particles when laser-induced gas breakdown is applied to energy addition around an aircraft. The thermal structure from laser-induced breakdown will be altered by the interaction with atmospheric

The original version of this chapter has been revised and is now available open access. A correction to this chapter is available at https://doi.org/10.1007/978-3-319-91017-8_149

T. Ukai $(\bowtie)$

Department of Mechanical Engineering, Osaka Institute of Technology, Osaka, Japan

e-mail: takahiro.ukai@oit.ac.jp

H. Zare-Behtash · C. White · K. Kontis

School of Engineering, University of Glasgow, Glasgow, UK

(C) The Author(s) 2019

A. Sasoh et al. (eds.), 31st International Symposium on Shock Waves 2, https://doi.org/10.1007/978-3-319-91017-8_2 
aerosols. There is a possibility that several plasma regions can be formed due to vaporization of the suspended aerosols. When considering interaction of a laser beam with a material, a vapor plume is formed due to vaporization from material melting, and the interaction between the vapor and the laser beam results in ionization of the gas [6]. The ionized gas can absorb laser energy; thereafter a shock wave induced by an absorption wave is generated [7]. The vapor-laser interaction is related to plasma formation as well as shock wave generation. It is expected that laser-particle interactions can lead to the generation of several plasma regions; thus a different shock wave structure would appear compared with gas breakdown in pure air.

In this study, the liquid impurity effect on laser-induced gas breakdown was experimentally investigated using flow visualization and numerically using threedimensional Navier-Stokes equations. This research focuses on the behavior of shock wave propagation and shock-shock interaction in laser-induced gas breakdown, rather than the plasma kernel dynamics.

\section{Experimental Setup}

A laser beam was focused in a quadrilateral stainless steel vacuum chamber with a volume of $0.138 \mathrm{~m}^{3}$. The chamber has a top quartz window of $98 \mathrm{~mm}$ in diameter and a pair of side quartz windows of $137 \mathrm{~mm}$ in diameter. The chamber was connected to a vacuum pump, and nitrogen gas was supplied until the pressure within the chamber recorded $100 \mathrm{kPa}$ after evacuating the air. The nitrogen gas was passed through a particle generator (TSI, model: 9307-6, $1000 \mathrm{~L} / \mathrm{min}$ of aerosol flow rate) to provide olive oil particles $1 \mu \mathrm{m}$ in diameter. The mixture of nitrogen gas with suspended olive oil was supplied to the chamber. When considering laserinduced gas breakdown without the liquid impurity effects, nitrogen gas is directly supplied to the chamber. A pressure sensor and k-type thermocouple were installed on the chamber. These signals were monitored using a data acquisition system (National Instruments Corp., model: NI-9205 for pressure monitoring and NI-9213 for temperature monitoring) driven by LabVIEW. In the present experiments, the pressure and the temperature in the chamber were $P_{0}=99.6 \pm 0.3 \mathrm{kPa}$ and $T_{0}=293.3 \pm 1.8 \mathrm{~K}$, respectively.

To induce the laser energy focusing in the chamber, a Q-switched pulsed Nd:YAG laser beam with a wavelength of $532 \mathrm{~nm}$ was employed. A concave lens with a focal length of $-50 \mathrm{~mm}$ expands the laser beam, and a second convex lens with a focal length of $150 \mathrm{~mm}$ collimates the laser beam. The laser beam then passes through the top window of the chamber. The collimated laser beam is focused in the chamber by a convex lens with a focal length of $100 \mathrm{~mm}$. The laser energy in the vicinity of the laser focal position was $188.0 \pm 1.9 \mathrm{~mJ} /$ pulse.

The laser-induced blast wave was visualized using high-speed schlieren photography with a standard Z-type optical arrangement. The schlieren system consists of a 450-1000 W continuous light source with an Xe-Hg arc lamp (Newport, model: 
66921), a condenser lens with a focal length of $70 \mathrm{~mm}$, an iris diaphragm, a pair of 203.3-mm-diameter concave mirrors with a focal length of $1829 \mathrm{~mm}$, a knife-edge, an imaging lens, and a high-speed camera HPV-1 (Shimadzu, special resolution, $312 \times 260$ pixels). The images were acquired at a frame rate of $500 \mathrm{kfps}$ with an exposure time of $1 \mu \mathrm{s}$.

\section{Computational Method}

The numerical work uses the finite-volume OpenFOAM software package. The compressible flow solver rhoCentralFoam was chosen. This is a three-dimensional, transient, density-based compressible Navier-Stokes solver based on the centralupwind schemes of Kurganov and Tadmor [8]; hence it is second-order accurate in space. In this work, a backward difference time scheme is utilized, and so the results are also second-order accurate in time. The gas properties are set to be those of nitrogen initially at a temperature of $293.3 \mathrm{~K}$ and a pressure of $99.6 \mathrm{kPa}$ to remain consistent with the experiments. Despite the large temperature increment introduced by the laser energy deposition, a constant viscosity of $1.76 \times 10^{5} \mathrm{~Pa} \mathrm{~s}$ and a constant specific heat at constant pressure $C_{p}$ of $1040 \mathrm{~J} / \mathrm{kg} / \mathrm{K}$ are used throughout the current work. Such simplifications have previously been shown to be acceptable for capturing the shock wave behavior in these kinds of problems [1].

The energy deposited by the laser beam increases the temperature and pressure of the gas on a small timescale. As such, the heat addition is assumed to take place at constant density. The temperature increase $\Delta T$ in the gas after the laser is fired is calculated as

$$
\Delta T=\frac{\Delta E}{\rho \cdot C_{V} \cdot V}
$$

where $\Delta E$ is the energy added by the laser pulse, $\rho$ is the ambient gas density, $C_{v}$ is the specific heat of the gas at constant volume, and $V$ is the laser perturbation focal volume, which, in the current work, is estimated from a size of plasma region in the experimental results at early times.

Any numerical cells that fall within the spherical radius of the laser perturbation focal volume have their temperature increased by $\Delta T$, and the pressure is increased by the same factor. Real gas effects, such as chemical reactions, due to this large increment in temperature are not considered in the current numerical work.

Once the simulation begins, the large gradients of temperature and pressure act as sources of acceleration on the right-hand side of the Navier-Stokes equations, converting the internal energy to kinetic energy and resulting in the formation of a shock wave that propagates outward. The numerical domain is axisymmetric, and all simulations in the current work use a variable time step with a maximum Courant number of 0.8 . 


\section{Results and Discussion}

Different shock wave formation/propagation are apparent in laser-induced gas breakdown with and without particles. Figure 1 shows experimental schlieren images of gas breakdown without particles. Rapid local heating due to the laser focusing results in plasma generation, which can be seen as the bright region in the schlieren images. A laser-induced blast wave also propagates spherically into the surrounding gas. A spherical laser-induced blast wave is observed at an elapsed time of $4 \mu \mathrm{s}$ in gas breakdown without particles. On the other hand, an elliptic shaped blast wave appears in gas breakdown with particles at the same time (Fig. 2).

Two bright regions related to plasma generation appear at an elapsed time of $8 \mu \mathrm{s}$ (Fig. 2c), which results in two laser-induced blast waves being produced in close proximity to one another. Thereafter, a longitudinal elliptic shaped blast wave is formed due to the interaction of the two laser-induced blast waves. It is expected that the laser beam with a narrow beam waist impinges on the particles at both the ideal laser focal position as well as the upper part of the ideal laser focal position. This results in the generation of two gas breakdown regions along the beam path. The location of the multiple laser focal points may depend on the particle density and laser energy level. The shock wave fronts that propagate toward the opposite laser focal point impinge one another; hence a transmitted shock wave (TSW) appears.

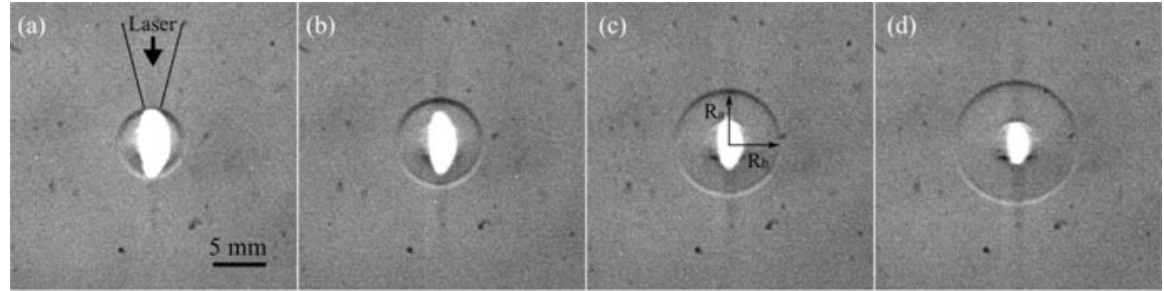

(a) (b) (c) (d)

Fig. 1 Experimental schlieren images of gas breakdown without particles. (a) $\Delta t=4 \mu \mathrm{s}$, (b) $\Delta t=6 \mu \mathrm{s}$, (c) $\Delta t=8 \mu \mathrm{s}$, and (d) $\Delta t=10 \mu \mathrm{s}$

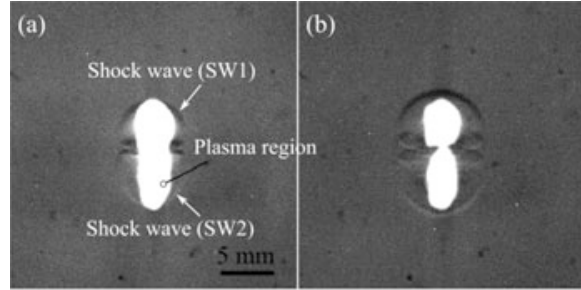

(a)

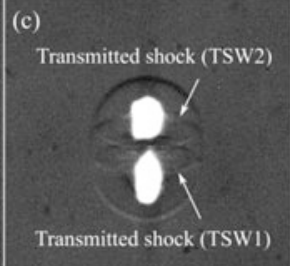

(c)

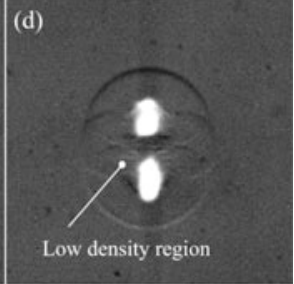

(d)

Fig. 2 Experimental schlieren images of gas breakdown with particles. (a) $\Delta t=4 \mu \mathrm{s}$, (b) $\Delta t=6 \mu \mathrm{s}$, (c) $\Delta t=8 \mu \mathrm{s}$, and (d) $\Delta t=10 \mu \mathrm{s}$ 


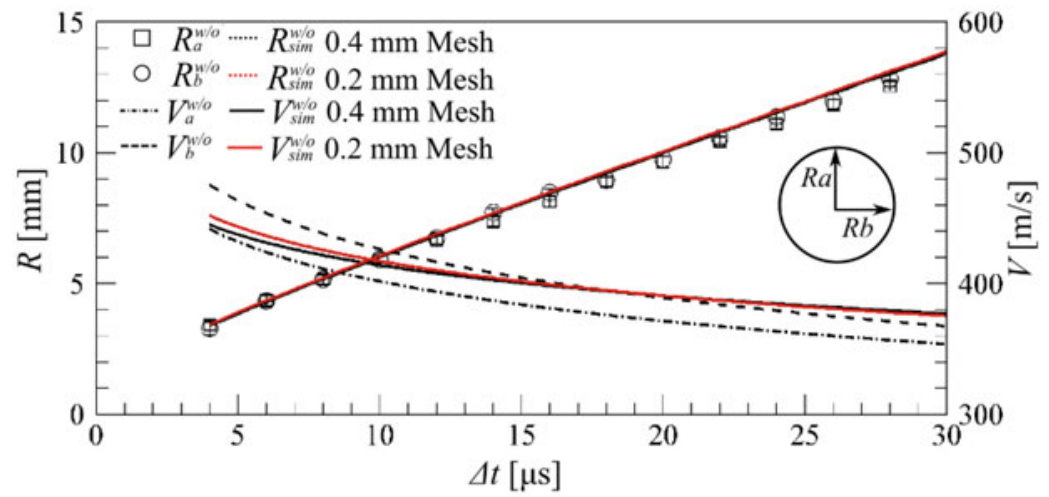

(a)

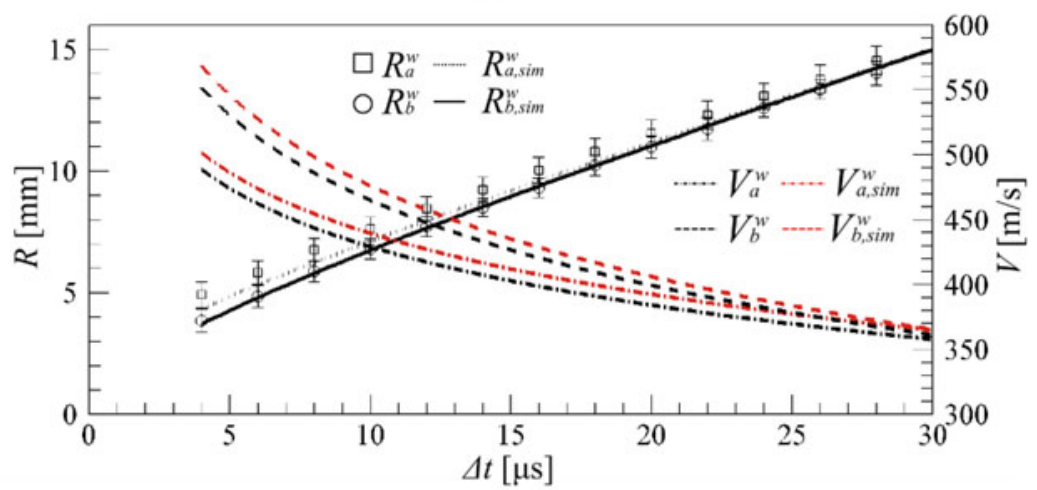

(b)

Fig. 3 Comparison of experimental and numerical shock velocities and radii. (a) Without particles. (b) With particles

The TSW interacts with the thermal heated region at the laser focal point, which results in distorting the spherical shape of the TSW. This is because the local shock Mach number decreases in the heated region.

The numerical results show good agreement with the experimental results for both gas breakdown with and without particles. In the gas breakdown without particles from experimental results (Fig. 3a), the longitudinal shock wave radius $R_{a}{ }^{w / o}$ is the same as the lateral shock radius $R_{b}{ }^{w / o}$. The error bars show the standard deviation from six repetitions. Additionally, the longitudinal shock wave velocity of $V_{a}{ }^{w / o}$ is similar to the lateral one $V_{b}{ }^{w / o}$. The velocities are obtained by logarithmic approximation. From the numerical results, both shock radius and shock velocity are in good agreement with the experimental results. There is little difference between the results with a cell size of $0.4 \mathrm{~mm}$ and $0.2 \mathrm{~mm}$, so the $0.4 \mathrm{~mm}$ size is used for the remainder of the numerical. 


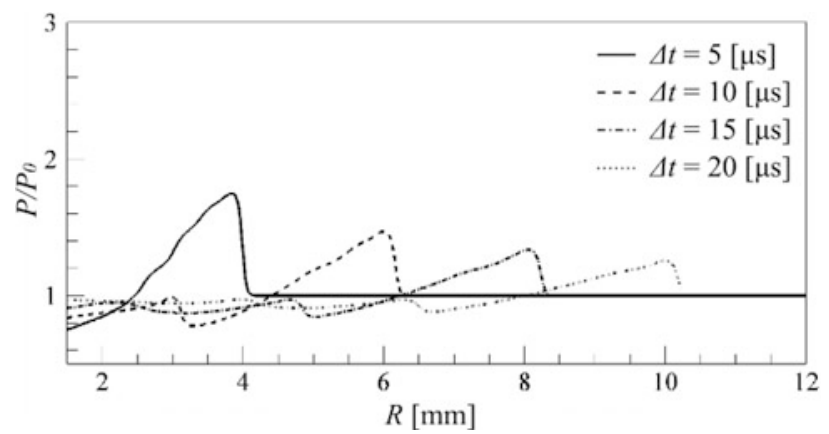

Fig. 4 Pressure history in gas breakdown without particles

From the experimental results of gas breakdown with particles, $R_{a}{ }^{w}$ is slightly larger than $R_{b}{ }^{w}$ because of the elliptic shaped blast wave; however, the longitudinal and lateral shock radii become the same with time. The error bars show the standard deviation due to 12 repetitions. In the numerical simulation, two heated spots at the same focal location as the experiments were used as the initial conditions. Since the numerical results show good agreement with the experimental results, it can be deduced that the hypothesis that the laser beam is focused at two different locations appears to be correct.

The shock wave generated in gas breakdown with particles is stronger than without particles. Figures 4 and 5 show the pressure history from the simulations, with and without particles, respectively. The pressure rise at the shock wave front is followed by an immediate decay to a negative value formed in the laser-induced blast wave. Then, the negative value induces a pressure rise so that pressure recovers to the atmospheric pressure. The time between the primary pressure rise and the pressure recover is almost the same, independent of the interaction with particles, but the pressure magnitude differs. The overpressure along the $\mathrm{x}$-axis is highest because the shock waves generated from the two focal points interact at this location, which results in shock focusing. This tendency is similar to the velocity history (Fig. 3) where the velocity $V_{b}{ }^{w}$ is the highest at an elapsed time of $5 \mu \mathrm{s}$.

In the pressure history along the y-axis (Fig. 5b), a second pressure rise at the shock front of the TSW appears. The simplification of the initially uniform high-temperature and high-pressure region in the simulations does not result in an accurate capture of the low-density bubble behavior; thus, the location of the second pressure rise, corresponding to the location of the TSW in Fig. 2, is not expected to correspond with the experimental result due to different shock-bubble interaction behavior. Future numerical work will focus on improving the low-density region behavior. For example, it is known that the heated region takes the form of a teardrop shape rather than a sphere [9]. 


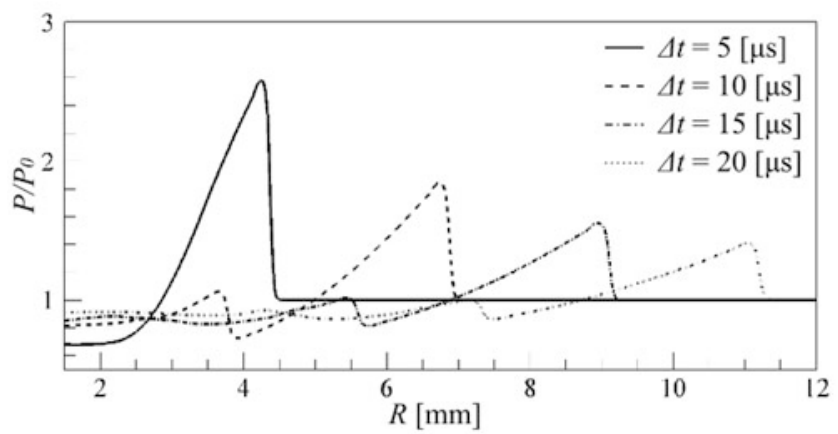

(a)

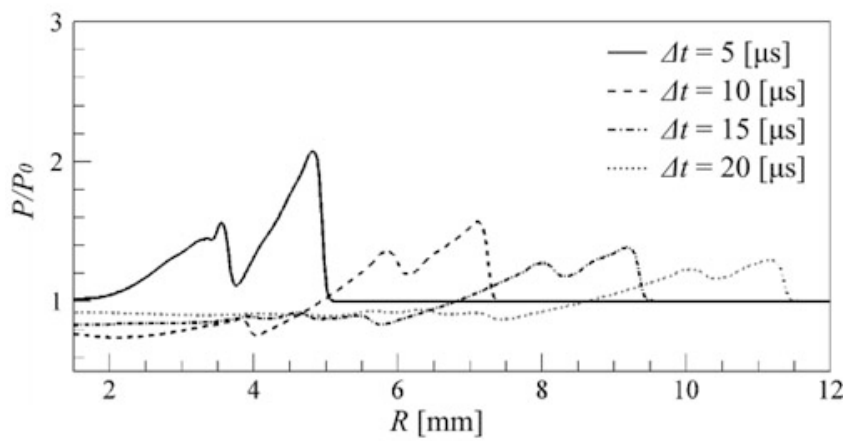

(b)

Fig. 5 Pressure history in gas breakdown with particles. (a) Along the x-axis. (b) Along the y-axis

\section{Conclusion}

This study experimentally and numerically investigated the liquid impurity effect on laser-induced gas breakdown in quiescent gas. When gas breakdown was induced with particles present, an elliptic shaped blast wave was generated, whereas a spherical blast wave was formed without particles at the same elapsed time. It is concluded that the presence of olive oil particles causes the laser beam to focus on more than one point, which results in the generation of multiple laser energy deposition zones along the beam path. In gas breakdown with particles, a larger overpressure magnitude appeared on the perpendicular axis of the laser path.

Acknowledgement We would like to dedicate this paper to Prof. Margaret Lucas at the University of Glasgow for her kind arrangement of the usage of the high-speed camera and to Dr. Richard Green at the University of Glasgow for setting up the laser facility. This research work was supported by European Commission, H2020-MSCA-IF (Project reference: 654318). The laser facility was supplied by National Wind Tunnel Facility project (EPSRC grant number: EP/L024888/1). 


\section{References}

1. H. Yan et al., AIAA J. 41, 10 (2003)

2. Y. Ogino et al., Phys. Fluids 066102, 21 (2009)

3. J.-H. Kim et al., AIAA J. 49, 9 (2011)

4. R. Kandala, G.V. Candler, AIAA J. 42, 11 (2004)

5. J.A. Syage et al., J. Appl. Phys. 64, 1499 (1998)

6. D. Bauerle, Laer Processing and Chemistry (Springer, New York, 2011), pp. 4-5

7. C. Boulmer-Leborgne, J. Hermann, B. Dubreuil, Plasma Sources Sci. Technol. 2, pp. 219-226 (1993)

8. C.J. Greenshields et al., Int. J. Numer. Methods Fluids 63, 1 (2010)

9. S. Ghosh, K. Mahesh, J. Fluid Mech. 605, pp. 329-354 (2008)

Open Access This chapter is licensed under the terms of the Creative Commons Attribution [4.0 International License (http://creativecommons.org/licenses/by/4.0/)], which permits use, sharing, adaptation, distribution and reproduction in any medium or format, as long as you give appropriate credit to the original author(s) and the source, provide a link to the Creative Commons licence and indicate if changes were made.

The images or other third party material in this chapter are included in the chapter's Creative Commons licence, unless indicated otherwise in a credit line to the material. If material is not included in the chapter's Creative Commons licence and your intended use is not permitted by statutory regulation or exceeds the permitted use, you will need to obtain permission directly from the copyright holder. 